Article

\title{
Determining the Optimal Configuration of the Multi-Ring Tree for Bluetooth Multi-Hop Networks
}

\author{
Chih-Min $\mathrm{Yu}^{1, *}$ (1) and Ting-Wei Hsu ${ }^{2}$ \\ 1 The Department of Electronics Engineering, Chung Hua University, Hsinchu 30012, Taiwan \\ 2 The Department of Electrical Engineering, Chung Hua University, Hsinchu 30012, Taiwan; \\ m10401023@chu.edu.tw \\ * Correspondence: ycm@chu.edu.tw; Tel.: +886-3-5186034
}

Received: 20 August 2017; Accepted: 31 August 2017; Published: 5 September 2017

\begin{abstract}
In this work, a multi-ring tree algorithm is proposed for Bluetooth low-energy networks with non-uniform distribution of devices. In a dense area, a leader root is elected during the leader election phase and a min-path algorithm is introduced to determine the optimal number of rings for various numbers of discoverable roots. According to the optimal configuration, the leader root connects to its one-hop neighboring roots to form the first-tier ring; each new root connects with one downstream root, and these roots connect together to form the second-tier ring until the desired outermost ring is reached. In sparse areas, each root constructs its own spanning tree subnet, finally creating a multi-ring tree scatternet. To achieve the least route discovery overhead, a multi-hop self-routing protocol is developed to efficiently deliver packets. Computer simulations show that the optimal multi-ring subnet can be determined; the optimal multi-ring tree outperforms conventional dual ring-tree (DRT) and Bluetooth hybrid ring-tree (BlueHRT) in terms of network performance.
\end{abstract}

Keywords: Bluetooth networks; scatternet formation; topology configuration; routing protocol

\section{Introduction}

Bluetooth is currently one of the most promising available ad hoc wireless connectivity technologies, and the introduction of its advantageous features is considered to be one of the main enablers of the Internet of Things (IoT) [1]. Bluetooth Low Energy (BLE) technology [2] can be embedded in existing mobile and portable devices, offering the advantages of a short-range radio, low power, and low-cost wireless connectivity. At present, globally there is wide use of smart devices such as smartphones, tablets, and laptops connected to multi-hop networks through BLE devices [3]. Many BLE data applications are emerging in daily life, such as in health networks [4], smart grid [5], wireless sensor networks [6,7], etc.

With integrating the latest innovative communications, smart grid applications become increasingly popular, as they aim to meet the energy efficiency requirements [8]. To improve the reliability and efficiency of power utilization, a smart grid is a combination of two-way data communications, distributed computing, and smart sensors. In addition, one application field is the home area network (HAN) [9], where a communication path is created among smart meters, home appliances, and plug-in electronic devices. Among wireless protocols in recent years, new technologies are emerging aiming at an energy-efficient communication. A novel research activity has focused on BLE because it is an appealing solution in becoming a key technology for smart homes in low-power, low-cost, and small devices $[5,10,11]$. However, these applications pose some important research challenges, such as how to connect BLE devices to share information among users, and how to form the desired network configurations for various types of applications.

Recently, the specifications of BLE 5 have been presented to offer significant enhancements that differ in range, speed, and energy consumption compared to the earlier versions of the protocol. 
Another main feature of BLE is that nodes can play dual roles as relays, and such relay nodes enable inter-piconet communication. The relay node design increases the possibility of implementing a scatternet formation algorithm and multi-hop routing for BLE devices. To-date, the development of multi-hop routing networks has faced some inherent technical challenges, including research design issues related to the formation algorithms [12] and the routing protocols $[13,14]$. The problem involves how to construct individual piconets and connect them together into a scatternet. In addition, the routing protocols must deal with the problem of delivering messages efficiently in such scatternets.

Many scatternet formation algorithms have been developed to construct a multi-hop ad hoc network, and they can be classified into two categories. One category-the single-hop scenario $[15,16]$ - deals with situations in which all nodes are within radio range. The other category—the multi-hop scenario $[17,18]$ —handles situations in which not all nodes are necessarily within radio range. Most of the current scatternets in the multi-hop scenario are generated in a distributed manner [19-22]. In addition, a number of different topology models [23] can be generated according to the desired purpose of the scatternet. Most of these scatternet models have been studied and can be generally categorized as tree-based, mesh-based, ring, or star topologies.

With a non-uniform distribution application, such as a conference hall or an intensive care unit in a hospital, Bluetooth hybrid ring-tree (BlueHRT) [15] forms a ring topology in one dense area, which is connected with multiple tree subnets around the other sparsely covered areas. However, this protocol partitions networks by collecting complete topology information in a coordinator, and involves considerable formation overheads. In addition, using a ring subnet as the core network design is not reliable enough because it is vulnerable to significant failure if a node in the ring subnet fails.

In [24], a dual-ring tree (DRT) protocol generates a dual-ring subnet in one dense area, which is connected to multiple tree subnets around the other sparsely covered areas. To consider the emerging probability of the multi-hop situation in the dense area, a distributed formation algorithm is presented to cope with the multi-hop instances, while a localized formation algorithm is proposed to cope with the pure single-hop instance. As a result, the DRT topology outperforms conventional BlueHRT in terms of network reliability and routing efficiency for Bluetooth networks.

The routing protocol design is another notable leading research issue for Bluetooth multi-hop scatternets [25]. Thus far, many well-known routing protocols have been presented, including proactive [15,18], reactive [26], and hybrid methods [27] for Bluetooth networks. To achieve the route discovery with the least overhead, a self-routing protocol is presented for both tree [28] and layer-ring topologies of the single-hop scenarios [29]. Currently, the multi-hop self-routing protocol remains as a research challenge.

To extend the DRT architecture into a generalized $n$-ring network solution, a multi-ring tree with a multi-hop self-routing protocol is proposed. In order to determine the optimal configuration of one dense area and many sparse areas, an optimal multi-ring tree formation algorithm is presented herein. During the leader election phase (LEP), a leader root is elected and then connects to its neighboring nodes as a root in order to build the multi-ring subnet, thus improving the connectivity of the core network in the dense area. Each root in the multi-ring subnet then starts to build its corresponding tree-shaped subnet around other sparse areas. Finally, a multi-ring tree topology is constructed.

The multi-ring design aims to determine the optimal configuration for various sizes of network topology. To achieve this goal, a min-path algorithm is introduced to determine the optimal number of rings $R$ and the best number of nodes in each ring $P$ for various numbers of nodes $N$. With a constraint criterion between $R$ and $P$, the resulting combination of $R$ and $P$ achieves the minimum hop length locally and constructs a more symmetrical multi-ring subnet. To efficiently deliver packets for both multi-ring and tree subnets, a self-routing protocol is an energy-efficient solution to achieve the least route discovery overhead for a multi-hop scenario.

The remainder of this paper is arranged as follows. Section 2 proposes the detailed operation of the multi-ring tree formation algorithm. Section 3 presents a self-routing protocol with a self-healing scheme to efficiently maintain the topology and deliver packets over the multi-ring tree network. 
Section 4 demonstrates the optimal configuration and routing performances via computer simulations. Finally, conclusions are drawn in Section 5.

\section{Formation Algorithm}

\subsection{Motivation of Multi-Ring Design}

It is assumed that a non-uniform environment is concentrated in a certain area with higher density, while the other extended areas are sparse areas. The high-density area belongs to the one-hop scenario and is designed as a multi-ring subnet. The other extended sparse areas belong to the multi-hop scene, which can be designed as a tree subnet. With the hybrid network design for the non-uniform scenario, the multi-ring tree benefits from both ring and tree network advantages.

To generate a multi-ring subnet, a coordinator discovers and configures neighbor information $N$ into the desired multi-ring subnet. The resulting multi-ring subnet can be the backbone network for the highly dense area. On the other hand, the discovered number of nodes $N$ can be a random variable for various scenarios. Therefore, how to plan $N$ into the optimal $R$-layer multi-ring architecture is an interesting research issue.

Two main situations are considered in the design method: the first is how to form the desired multi-ring subnet, and the second is how to achieve the optimal configuration with the minimum multi-ring path length. Because $N$ is a variable number, it can be divided into two general cases: prime number and composite number cases. When $N$ is a prime number, $N$ decreases by 1 until it is decomposable, since the prime case cannot form a multi-ring. When $N$ is decomposable, $N=R \times P$, where $R$ is defined as the number of rings and $P$ is defined as the number of nodes in each ring. In addition, to minimize the average path length of the multi-ring network, designing the symmetry multi-ring configuration to make the value of $R$ and $P$ as close as possible is intuitive.

When $N$ is decomposed into $R$ and $P$, it is found that some $N$ have many combinations of $R$ and $P$. Therefore, all possibilities of combinations need to be calculated, and the corresponding average path lengths must be compared to determine the optimal configuration of $N$. To make the multi-ring close to a symmetrical configuration, it is better to make $R$ and $P$ values closer in the multi-ring design. As a result, a constraint is proposed to limit the difference between the $R$ and $P$ to make the resulting network more symmetrical. At the same time, the constraint design also reduces the computational complexity of all possible combinations to more quickly determine the desired configuration.

In the example of $N=48$, there are eight decomposable multi-ring combinations except the cases $(24,2)$ and $(48,1)$ since at least three nodes are required to form a ring subnet. After calculating the various cases, the path length result of each combination is shown in Figure 1, which shows a minimum point when $R=6$ with $P=8$; this case achieves the minimum path length compared to all the other combinations. At the same time, the number of computations can be effectively reduced from eight to two if the constraint of $R-P$ is set to four, thus improving the computation time required to determine the best configuration.

Based on the above design points, we aim to develop a min-path algorithm to effectively determine the optimal multi-ring configuration. With an arbitrary given $N$ and $|P-R| \leq k$ constraint in the min-path algorithm, the system model can be formulated to quickly compute and search for the minimum point of the path length performance, and the most suitable $R$ and $P$ can be effectively determined. 


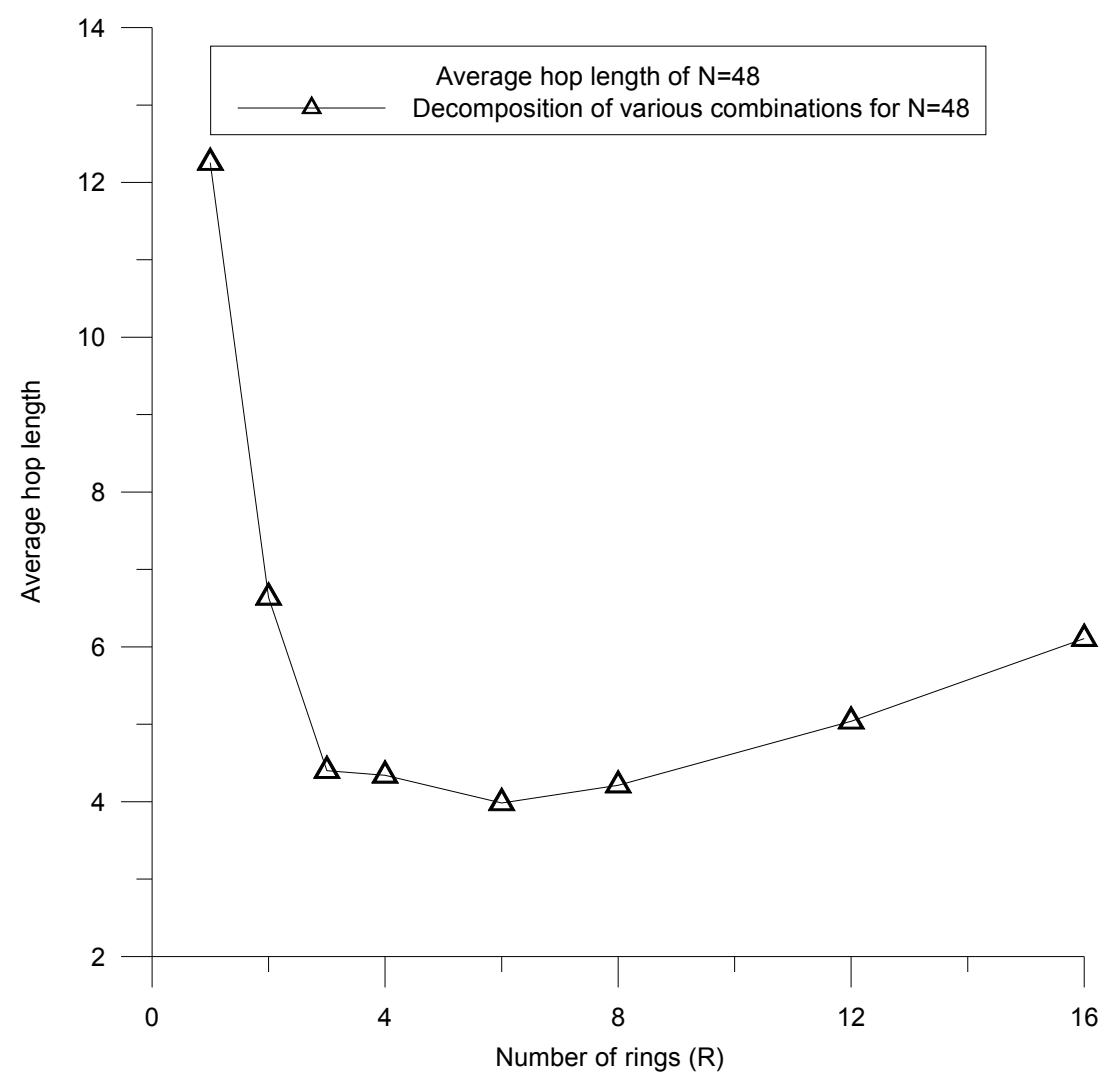

Figure 1. The average path length of $N=48$ example.

\subsection{Multi-Ring Tree Formation Algorithm}

\subsubsection{Min-Path Algorithm}

In order to improve the topology's reliability, other than the conventional single-ring network, this work proposes a multi-ring tree scatternet formation algorithm. The multi-ring subnet as a core network design effectively improves network reliability. To effectively form the desired topology, the algorithm discovers the topology only in the dense area instead of the global area as the conventional single-ring network does, in order to reduce the computation and communication complexity. In the dense area, a min-path algorithm is also presented to determine the optimal configuration of the multi-ring subnet.

During the node discovery phase (NDP) [30], each node alternatively operates as a scanner or an advertiser by a scan probability $p$. In the multi-ring tree, a node plays a scanner if its probability $p$ is greater than 0.5 , or as an advertiser otherwise. In a time interval $T$, each scanner increases its neighbor count by one when receiving an advertising message from a new neighbor. After the completion of NDP, a leader election phase (LEP) is started afterward. When two nodes meet, the node with the higher neighbor count value wins the role as a master and the other one plays a slave. During the connection, each slave sends its collected neighbor information to the master and stops to advertise message. When two nodes have the same neighbor count value, the node with the lower ID becomes the master. In this way, the masters continue to scan neighbor information and compare their neighbor count values by exchanging advertising messages. At the completion of LEP, the last master is elected as a leader root with the maximum neighbor count value in the dense area.

At the end of LEP, a leader root discovers the local topology with $N$ roots' information in the dense area. The min-path algorithm is introduced here to determine the local optimal topology of the multi-ring subnet. In the algorithm, the input parameter is $N$, and the output parameters are the number of rings $R$ and the number of nodes in each $\operatorname{ring} P$, where $N$ can be factorized into the $R$-ring 
and $P$-node in each ring. The minimum value of $R$ and $P$ is 1 , and the maximum value of $R$ and $P$ is $N$. The decision criterion is compared by the average hop length $H$, where $H_{i}$ is a function of $R$ and $P$ for a given combination $i$, where $m$ is the maximum value of $i$. When many decomposable combinations $i$ exist for any given $N$, the minimum point needs to be discovered by comparing the average hop length $H_{i}$. To minimize the computation complexity of $H(R, P)$, the constraint criterion $|P-R| \leq k$ is also introduced, where $k$ is a constant; the smaller $k$ reduces the computation complexity of the min-path algorithm. The min-path and the constraint criteria can be expressed as Equations (1) and (2), respectively.

$$
\operatorname{Min}_{i=1 \ldots m}\left(H_{i}\left(R_{i}, P_{i}\right)\right)
$$

Subject to:

$$
\left|P_{i}-R_{i}\right| \leq k
$$

The smaller $k$ for each $N$ intuitively brings the $R$ closer to the $P$. The resulting topology is more approximate to the symmetry deployment. However, when $N$ is not decomposable, $N$ decreases by 1 until the value can be factorized and the $|P-R| \leq k$ constraint is satisfied. On the other hand, when $N$ is decomposed into $R$ and $P$, if $|P-R|$ does not satisfy the constraint, then $N$ decreases by 1 until it can be decomposed into $R$ and $P$. Finally, the optimal configuration with the least hop length can be decided, and the suitable combination of $R$ and $P$ can be found. In addition, the closer $R$ and $P$ can satisfy the $|P-R|$ constraint, the better is the formation of a more symmetrical multi-ring configuration.

When $N=34$ and the constraint $|P-R|=4, N$ can be divided into $(R=1, P=34)$ and $(R=2$, $P=17)$; the resulting $R$ and $P$ sets do not meet the constraint. Next, $N$ decreases by 1 into 33 with $(R=1, P=33)$ and $(R=3, P=11)$ sets, which do not satisfy the constraint. Here, $N$ decreases by 1 again until the constraint is satisfied by two computed sets: $(R=4, P=8)$ and $(P=8, R=4)$. As a result, the optimal configuration can be determined by comparing the average hop length of $(R=4, P=8)$ and $(P=8, R=4)$; the resulting configuration is $(R=4, P=8)$.

\subsubsection{Multi-Ring Tree Formation Algorithm}

After determining the local optimal configuration with suitable $R$ and $P$, the leader root connects its one-hop neighboring nodes to form the multi-ring tree scatternet. The neighboring nodes can then be regarded as the first-tier new roots, and the leader root connects them together into the first-tier ring subnet. The number of roots in the first-tier ring is $P$. Once the first-tier ring subnet is formed, each root at the ring subnet connects to one neighboring node as the second-tier new root. The roots at the first-tier ring propagate their second-tier new roots' information and instruct them to connect with each other to form the second-tier ring subnet. In this way, the third-tier roots are discovered and connected until the desired R-tier ring subnet is achieved. As a result, a multi-ring subnet is constructed, and the bridge node can be a master/slave or a slave node.

Using $N=17$ and $|P-R| \leq 2$ as an example in the dense scenario, a leader root R1 discovers 16 other neighboring nodes. The min-path algorithm is executed by the coordinator R1, and $N$ is decreased by 1 into 16 since the initial $N$ is a prime number. The leader root then computes all possible $(R, P)$ sets, including $(1,16),(2,8),(4,4)$, and compares them with the constraint. As a result, the $(4,4)$ set satisfies the constraint and the optimal configuration is determined.

After determining the optimal configuration with $R=4$ and $P=4$, the leader root assigns the identification sequentially from R2 to R16 for all other roots and computes the connection information for each root. Here, R1 propagates the connection information for root candidates of the first-tier ring R2, R3, and R4, as shown in Figure 2a. The first-tier ring is formed by connecting R1, R2, R3, and R4, as shown in Figure $2 \mathrm{~b}$. Each root in the first-tier ring then connects with its downstream root and propagates the connection information for them to form the second-tier ring, as shown in Figure 2c. In this way, the 4 by 4 multi-ring is generated until the outermost 4-ring is reached, as shown in Figure 2d. Figure 2 illustrates the formation process of the optimal multi-ring subnet in the dense scenario. 


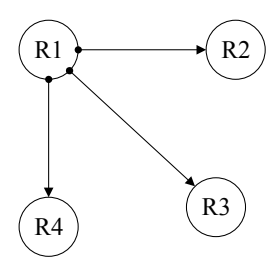

(a)

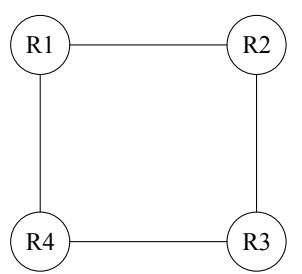

(b)

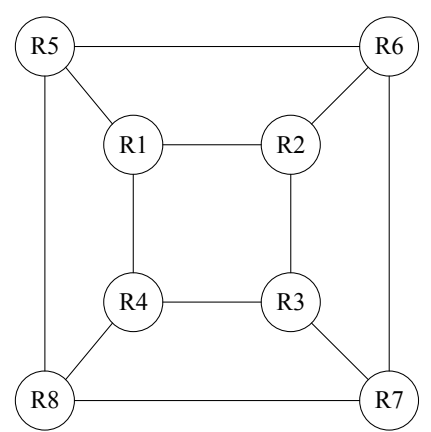

(c)

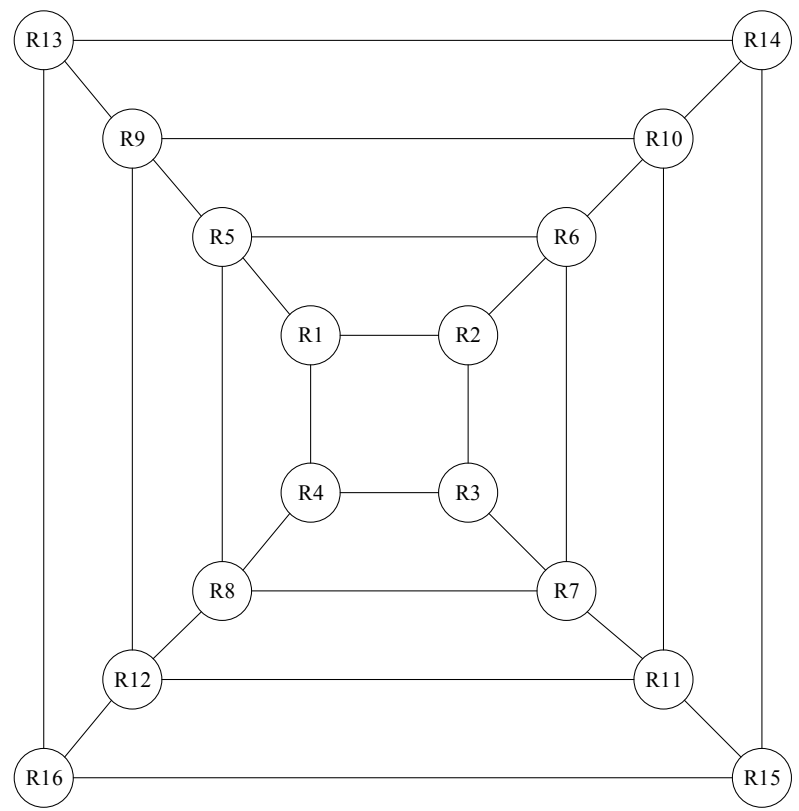

(d)

Figure 2. Formation example of an optimal multi-ring subnet: (a) R1 propagates the connection information for R2, R3 and R4; (b) The first-tier ring is formed by connecting R1, R2, R3 and R4; (c) Each first-tier root connects with its downstream root to form the second-tier ring; (d) The 4 by 4 multi-ring is generated until the outermost 4-ring is reached.

After the multi-ring subnet is generated, each new root in the multi-ring serves as a coordinator and begins to scan its neighboring advertising message and connects them to form their own piconets. The connected slaves then switch their roles to masters (called M/S nodes), and each M/S node repeats the same scan procedures as the root, while also connecting its advertisers to form its own piconet. This procedure is performed iteratively until the leaf nodes of the tree are reached. An illustrated example of the multi-ring tree scatternet with $N=16, R=4$, and $P=4$ is shown in Figure 3. In addition, the pseudo code of the multi-ring tree scatternet formation algorithm is listed in Algorithm 1. 


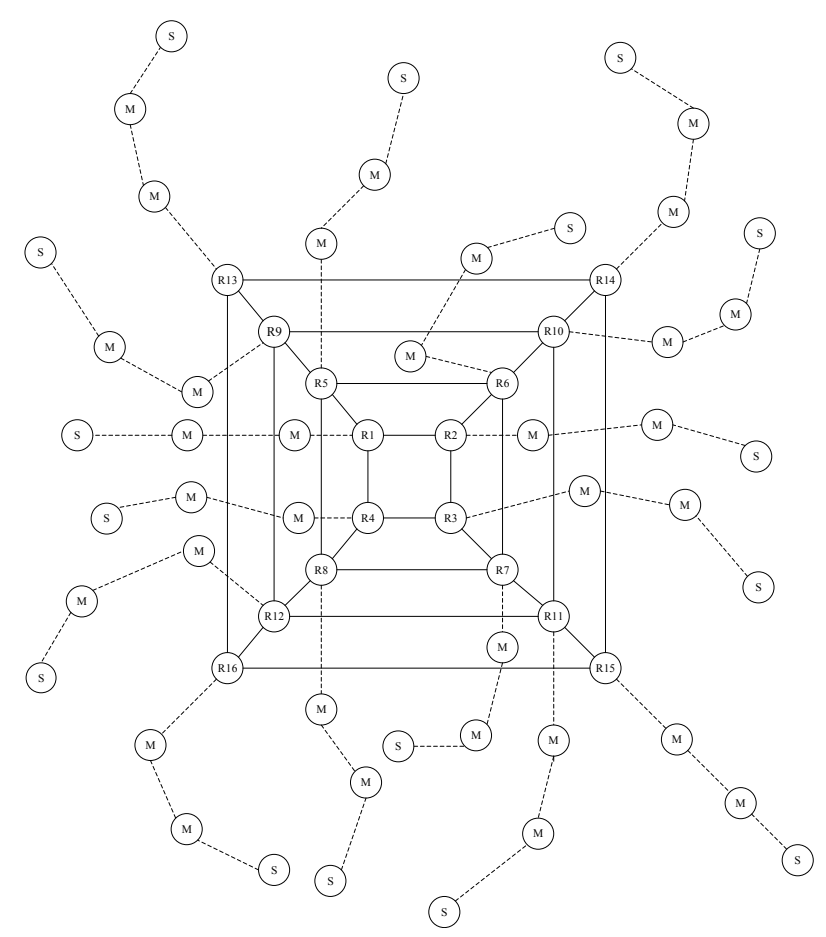

Figure 3. An illustrated example of the multi-ring tree network.

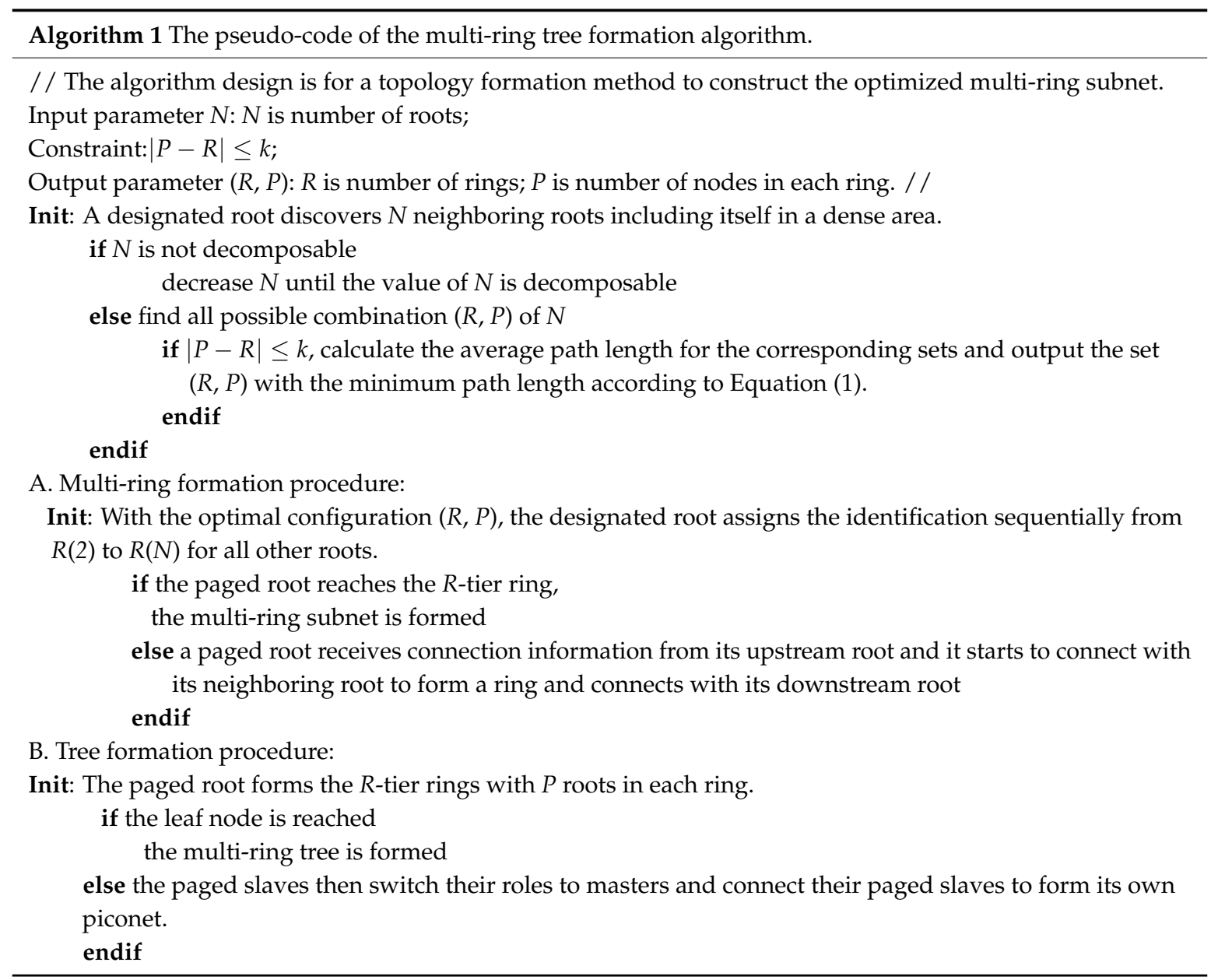




\section{Routing Protocol}

\subsection{A Self-Routing Algorithm}

In order to effectively deliver a packet in the multi-ring tree scatternet, this work also presents a self-routing protocol with a shortest path criterion. The self-routing algorithm for one single-hop with multi-hop scenarios can achieve the least overhead route discovery compared to the other traditional routing protocols. Figure 4 shows a packet address field of the multi-ring tree. The routing protocol designs three types of scatternet address field: the Ring-ID, Root-ID, and Tree-ID in the packet header to achieve the self-routing capability. The packet field of Ring-ID with $\left[\log _{2}^{R}\right]$ bits serves to specify the number of rings in the multi-ring subnet. The packet field of Root-ID with $\left\lceil\log _{2}^{P}\right\rceil$ bits addresses the number of roots in each ring. The Tree-ID defines all the downstream nodes in each root. In addition, the slave node is addressed by the Bluetooth AM_ADDR in a piconet. With these three types of address, each node can deliver packets to the desired destination node in a multi-ring tree network.

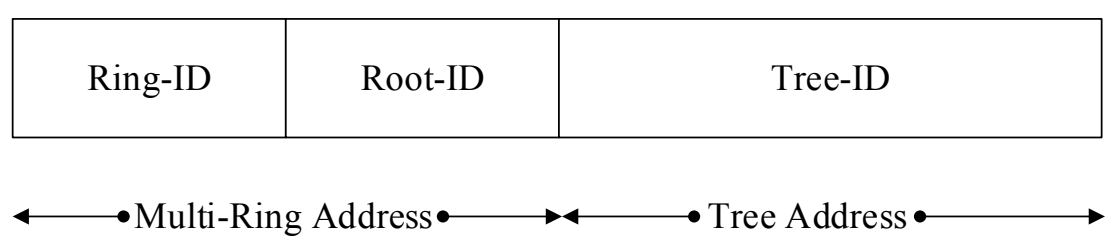

Ring-ID: The address for the number of rings

Root-ID: The address of roots in each ring

Tree-ID: The tree routing address

Figure 4. Multi-ring tree scatternet address field of a packet.

During the scatternet formation, a two-stage addressing method for the multi-ring tree is proposed. The first stage is to define the address for the multi-ring, and the second stage is to define the spanning tree address for the self-routing protocol. In the first stage, the Ring-ID is set to 1 for the roots in the first-tier ring, and the Root-ID is set to 1 for the leader root. In this way, the Ring-ID is set to 2 for the roots in the second-tier ring, the Ring-ID is set to 3 for the roots in the third-tier ring, and finally the outermost ring Ring-ID is set as $R$. At the same time, the Root-ID of roots in each ring is set by a constant counter $r$, where $1 \leq r \leq P$.

The second stage presents a tree-addressing criterion specifically to all nodes in each spanning tree for the multi-hop scenario. Each root with Tree-ID $=0$ connects with its downstream nodes with Tree-ID $=1$, and the Tree-ID sequentially increases by 1 until 7 for the most connected nodes in the first-tier tree. The master in the first-tier with Tree-ID $=1$ then connects with its downstream layer nodes with Tree-ID from 8 to 14, and the master Tree-ID $=2$ assigns its connected nodes with Tree-ID from 15 to 21 . In this way, the master with Tree-ID $=8$ connects with its downstream nodes and assigns the third-tier nodes Tree-ID from 57 to 63 , so that each upstream master can forward the packet to the destination node according to the Tree-ID. In summary, the address range of Tree-ID in the each-tier tree is assigned with the tree-addressing criterion by its parent with pTree-ID from $(7 \times \mathrm{pTree}-\mathrm{ID})+1$ to $7 \times($ pTree-ID +1$)$.

With the destination Tree-ID $=24$ as an example, the root of the destination receives the packet and computes the ID $=24$ by the tree-addressing criterion. The destination is located at the address range of its third connected master, and the packet will be forwarded to the parent master of the destination. The parent master in the first-tier then forwards the packet to the destination node, since ID $=24$ is the directly connected node of this master. 
To reduce the packet length of a larger ring subnet and efficiently deliver packets, a shortest path criterion is defined as Equation (3) to minimize the hop distance between root $i$ as $r_{i}$ and root $j$ as $r_{j}$. When a root in the ring subnet receives a new packet either from the tree subnet or the ring subnet, the Root-ID of the destination root will be checked with its own Root-ID by Equation (3). If $j$ is greater than or equal to $i$ and Equation (3) is satisfied, then the packet will be forwarded in a clockwise direction in the ring subnet; otherwise, the packet is forwarded in a counterclockwise direction. If $j$ is less than $i$ and Equation (3) is satisfied, then the packet will be forwarded in a counterclockwise direction in the ring subnet; otherwise, the packet is forwarded in a clockwise direction.

$$
\left|r_{j}-r_{i}\right| \leq P / 2
$$

The packet routing of the multi-ring tree network is generally classified into two cases: the intra-root and the inter-root routing. In the intra-root subnet routing case, a source node and a destination node are located at the same tree subnet under a root; the addresses of the Ring-ID and Root-ID in the packet header are the same. The destination of the root then computes the location of the destination according to the Tree-ID and forwards it to the destination via the resulting paths of the tree-addressing criterion.

In the inter-root subnet routing case, the packets are first forwarded from the tree subnet to the root of the source node. The source root $r_{s}$ then checks the Ring-ID and delivers packets to the ring layer of the destination root by the Ring-ID. After the packets are delivered to the ring layer of the destination, the packets are propagated to the root of the destination $r_{d}$ according to Equation (3). Finally, the destination root $r_{d}$ transmits the packets downstream to locate the destination node by the intra-root routing method when the destination is in its spanning tree subnet. As a result, the scatternet self-routing protocol of the multi-ring tree can be achieved by the proposed addressing method. In addition, the pseudo code of the multi-ring tree self-routing algorithm is listed in Algorithm 2.

\subsection{A Self-Healing Scheme}

A self-healing protocol can be achieved, since the multi-ring tree address scheme enables the maintenance of self-routing networks. This work considers four cases in the proposed self-healing protocol: a new node joining the network, a slave node failure, a master failure, and failure of one or two roots. When a node joins or fails in a piconet, each master adds a new AM_ADDR address for the new slave or deletes the slave connection from its piconet list, respectively. If a master/slave node leaves, the first slave node with the smallest AM_ADDR address becomes the new master; it connects all the other slaves with the same ring-tree address. In addition, the new master provides an update status for its affiliated root node.

When a root node in a ring subnet leaves, the slave node with the smallest AM_ADDR address in the piconet becomes a new root and sends out a root update message to its affiliated slaves and its directly connected neighboring roots. The topology maintenance for a single failed node can be applied for cases with two failed roots, since two failed roots are disconnected independently. For example, in Figure 3, if root R10 fails, then the candidate slave with the capability to connect with current directly connected roots in the R10 piconet becomes a new root, and the new R10 sends a root update message to its directly connected roots R6, R9, R11, and R14. When R1 and R6 both fail, their slaves become the new roots independently and coordinate their corresponding slaves. As a result, the multi-ring subnet still can be maintained locally. 


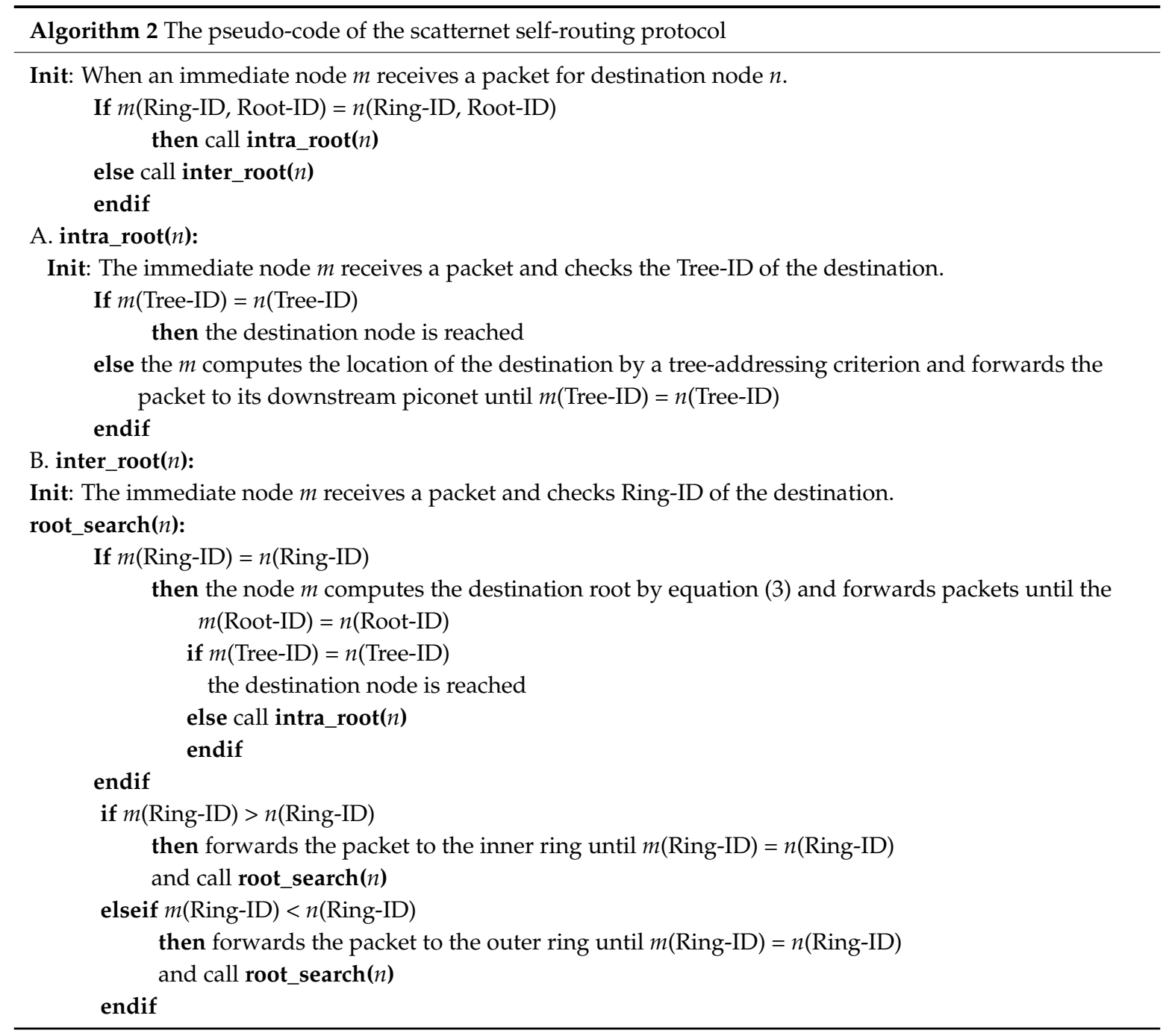

\section{Network Performance}

\subsection{Optimization Configuration of the Multi-Ring}

With $N$ ranging from 3 to 100, all factorization cases were calculated to determine the optimal number of ring $R$ and its corresponding number of nodes in each ring $P$ for the multi-ring subnet. In order to cover the computation nodes from 3 to 8 , the least constraint value of $\mathrm{k}$ is equal to 2 . In the single-ring subnet, the resulting $R$ is always equal to 1 , and $P$ is equal to $N$.

With $k$ varying from 0 to 3 , Figure 5 shows the performance of the optimal number of ring $R$ for various $N$. The $k=3$ case achieves the worst performance compared to the other three cases. In addition, the $k=0,1$, and 2 cases achieve the same performance concerning the optimal number of rings.

The number of nodes in a ring $P$ is determined by the number of nodes $N$ over the number of rings $R$, and thus it is inversely proportional to $R$. Figure 6 shows that the performance of the number of nodes in ring $P$ increases as the number of $\mathrm{k}$ increases for various $N$, and $\mathrm{k}=0$ achieves the least value of $P$. In the $k=0$ case, $R$ is equal to $P$, and this case achieves the optimal configuration among all other cases. However, it took more search cases than all other cases to locate the optimal $R$ and $P$ for various $N$, as shown in Figure 7 , and the single-ring case performs with the least computation overhead. The computation overhead is defined as the number of search times for a random input $N$ to be decomposable according to the constraint $k$ and to locate the optimal number of ring configurations. The number of search cases decreases as $k$ increases. As seen from the simulation, a trade-off exists between the computation overhead and the set value of $k$. 
With the min-path algorithm, the optimal multi-ring configuration can be determined and the corresponding $(R, P)$ sets with minimum path length generated. Figure 8 shows the average path length of the multi-ring for various $N$, and the $k=0$ case achieves the shortest path length compared to the other two cases. The $k=2$ case achieves almost the same hop length performance as $k=0$, and both cases achieve superior hop length performance compared to the single-ring case. In addition, the increment slope of the average hop length for the min-path algorithm is much smaller than the slope of the single-ring case increases, because the near symmetrical deployment of the multi-ring subnet can be achieved under the constraint $k$.

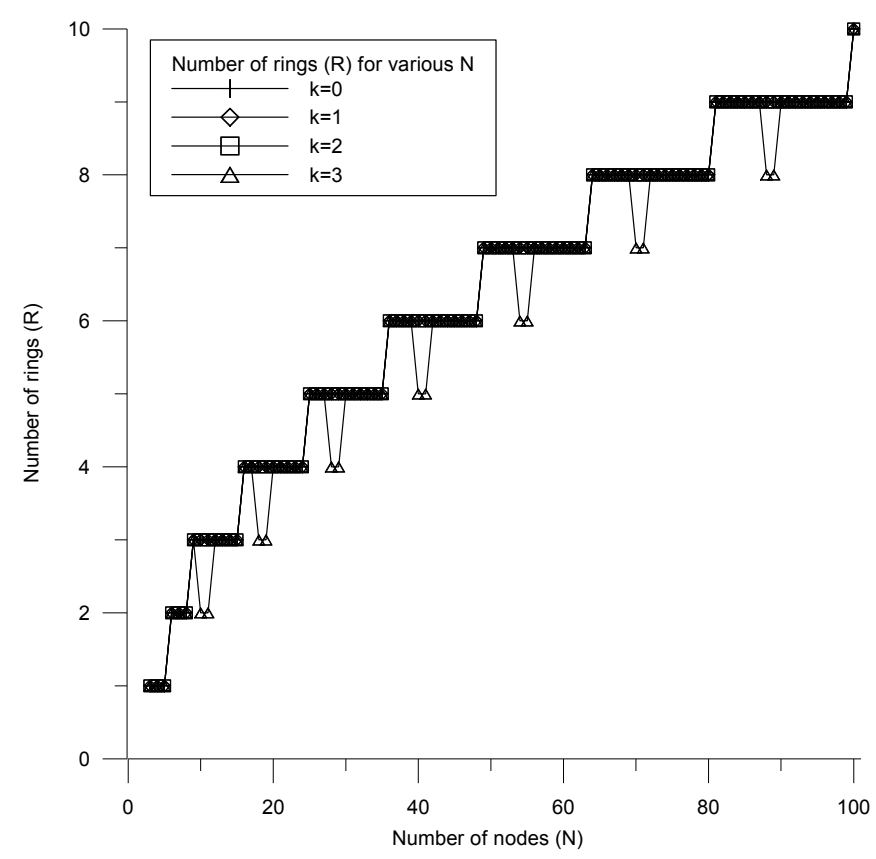

Figure 5. Optimal number of rings for various $N$.

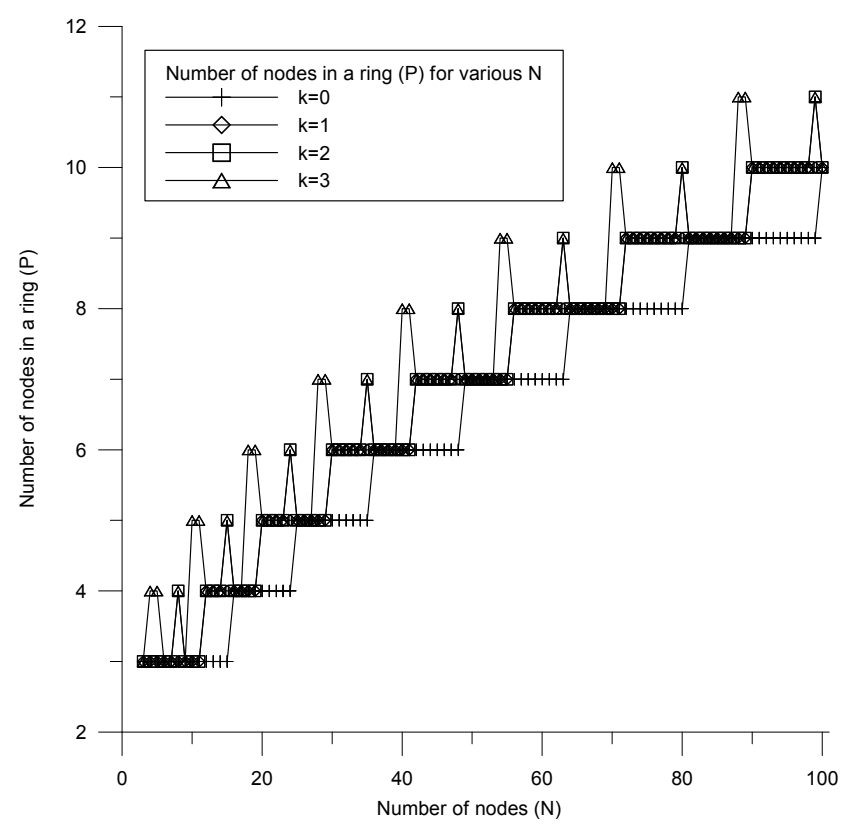

Figure 6. Optimal number of nodes in a ring for various $N$. 


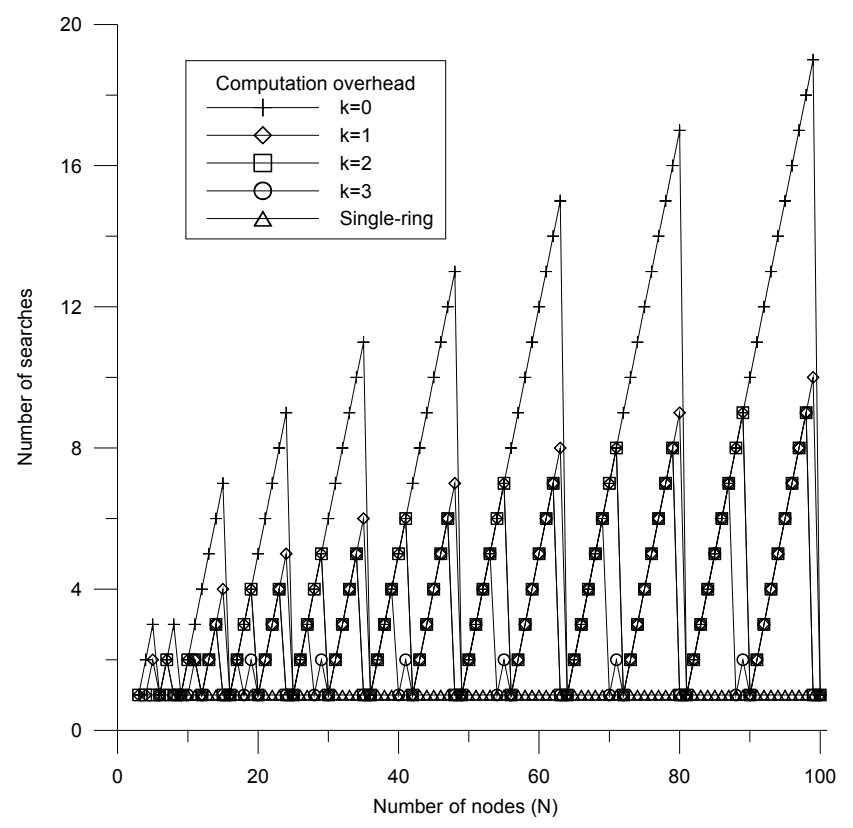

Figure 7. Computation overhead of min-path algorithm for various $N$.

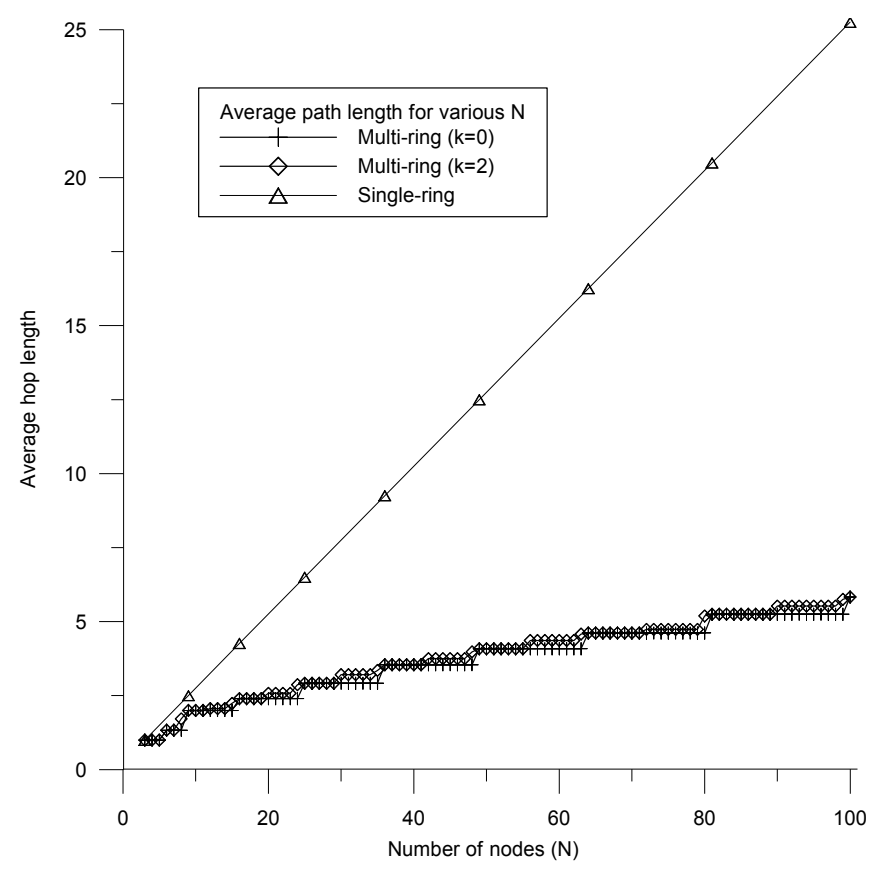

Figure 8. Average hop length of min-path algorithm for various $N$.

\subsection{Network Performance}

The simulation parameters for network performance are defined as follows. For the packet transmission of each node, each packet is generated in accordance with a Poisson arrival pattern. In addition, it was assumed that a single packet is sent in each routing session, and that each data packet has a duration of five time slots. A FIFO (First Input First Output) queue is provided with a length of 80 packets for each node. In each routing session, the source-destination pair was randomly selected, the time division multiplexing (TDD) scheme was adopted for both piconet and scatternet scheduling, and the packets were forwarded using the multi-ring tree topology with the self-routing protocol. For the core network topology, the multi-ring was simulated by $N=24$ roots with 76 nodes 
in the tree subnet. In addition, six combinations for a 24-root example were evaluated, including the single-ring case as the BlueHRT [15] with 24 nodes in a ring, a 2-ring case as the dual-ring tree (DRT) with 12 nodes in a ring [24], a 3-ring case with 8 nodes in a ring, a 4-ring case with 6 nodes in a ring, a 6-ring case with 4 nodes in a ring, and an 8-ring case with 3 nodes in a ring, which were simulated. In order to investigate the scatternet routing performance, three scatternet protocols-BlueHRT, DRT, and optimal multi-ring tree (Optimal MRT) - were compared. The BlueHRT and DRT protocols were chosen for comparison with the Optimal MRT because they both execute architectures similar to the multi-ring tree.

To estimate the impact of network congestion in a scatternet, a packet drop probability (PDP) metric was used. When the FIFO buffer overflows in each node, the damaged routing packets-including both the currently received and newly generated routing packets-are dropped. The PDP is defined as the ratio of the total number of dropped packets over the total number of generated packets for all nodes. Figure 9 shows the PDP of the six schemes, where the 4-ring case as the Optimal MRT achieved the lowest PDP compared to both BlueHRT and DRT cases. In addition, all the multi-ring tree simulation cases achieved better PDP performances than the traditional single-ring case in terms of BlueHRT. From the simulation results, it is clear that the roots and all other masters began to drop packets when the FIFO buffers overflowed.

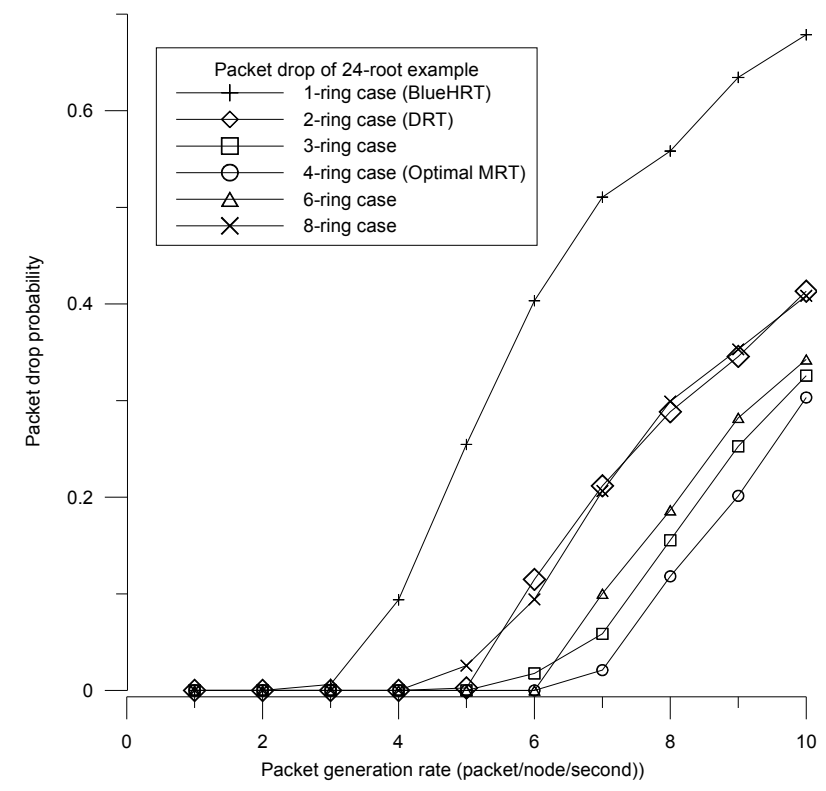

Figure 9. Packet drop probability of the Optimal MRT, DRT, and BlueHRT.

To reflect the scatternet capacity of the multi-ring tree, a packet throughput was used to evaluate network performance. The average packet throughput is defined as the ratio of the total number of successful routing packets over the total simulation time in seconds. The simulation results of the packet throughput for the 24-root of the multi-ring tree are shown in Figure 10. According to the simulation results, the 4-ring case as the Optimal MRT achieved the best throughput performance compared to the other five schemes, since the min-path algorithm can determine the optimal configuration with the minimum average path length for the multi-ring subnet. In addition, the routing packet throughput increased continuously as the packet generation rate increased, because the self-routing protocol combined with the multi-ring core network greatly improved the network routing performance compared to the BlueHRT and DRT cases. When the PDP starts as packet generation rate increases in Figure 9, the packet throughput almost reaches constant performance in Figure 10 and the network begins to be congested. As a result, the constant packet throughput can be regarded as the upper bound of network capacity for the multi-ring tree. 


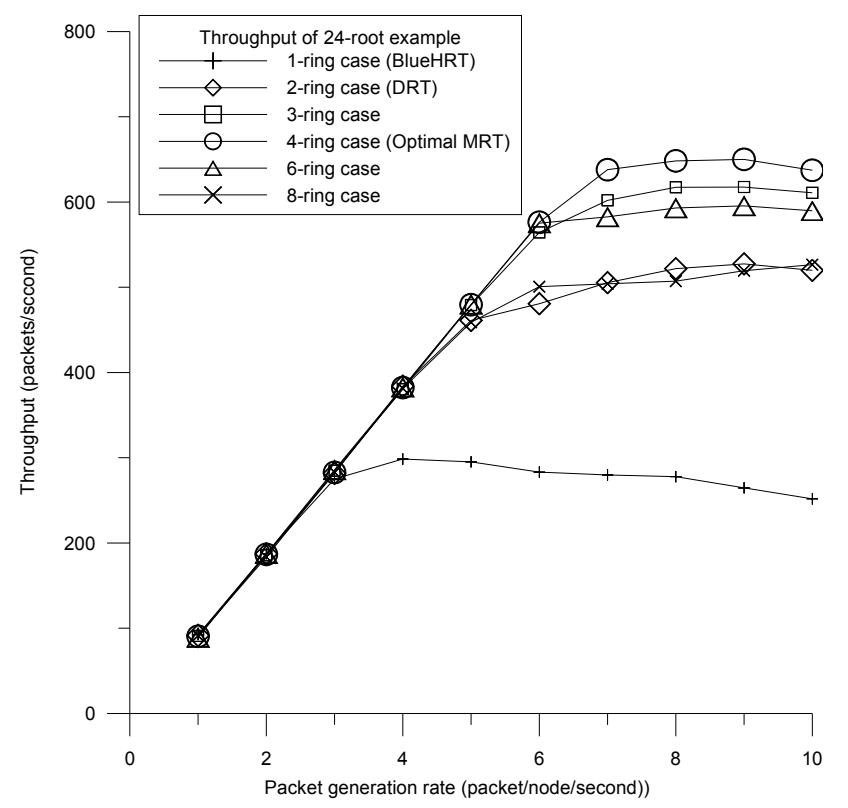

Figure 10. Throughput performance of the Optimal MRT, DRT, and BlueHRT.

In order to evaluate the multi-hop routing performance, an average packet delay metric was adopted to measure the end-to-end delay of a scatternet. The average packet delay of each routing packet is defined as the average packet transmission time from the first transmitted bit at the source node to the last received bit at the destination node. Figure 11 shows the average packet delay performances of the 24-root of the multi-ring tree. The Optimal MRT case generated the smallest average delay in the simulated six cases when the packet generation rate was smaller than six, as the Optimal MRT generated the shortest path connectivity. In addition, the average packet delay increased quickly as the packet generation rate rose for each case, and the network experienced a saturation phenomenon when network congestion occurred for all cases. The saturation phenomenon decreased the end-to-end packet delay when the PDP started to increase in Figure 9.

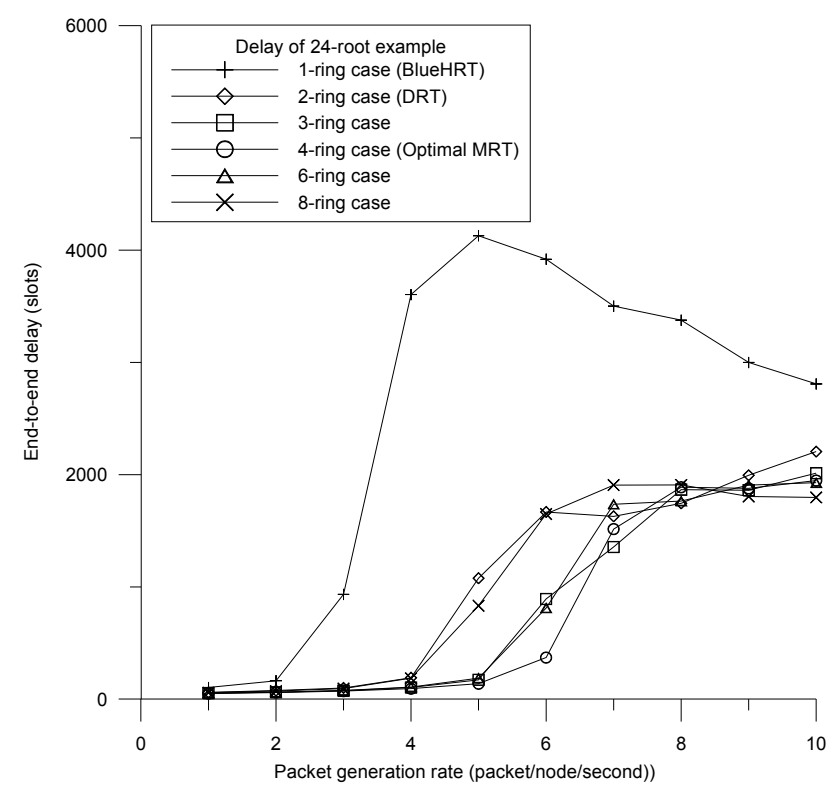

Figure 11. Average packet delay of the Optimal MRT, DRT, and BlueHRT. 


\section{Conclusions}

In order to determine the optimal configuration in one dense area and many sparse areas, this study proposes a multi-ring tree formation algorithm. With local topology information, the algorithm designs two stages to form the multi-ring tree scatternet. In the first stage, a leader root is elected and the optimal multi-ring configuration can be determined by a min-path algorithm for various sized subnets. In addition, a leader root connects with its neighboring roots to build the multi-ring subnet for the dense areas. In the second stage, each root in the multi-ring subnet starts to build its corresponding tree-shaped subnet for the other sparse areas. Finally, an optimal multi-ring tree topology is generated for non-uniform distribution applications. In addition, a proposed self-routing protocol for both multi-ring and tree topologies efficiently delivers packets over the scatternet, and this study also designs a self-healing routing protocol to extend network routing capability. As a result, the optimal configuration of the multi-ring subnet can be determined, and the multi-ring tree method outperforms conventional BlueHRT and DRT in terms of network performances. Thus, the optimal multi-ring tree topology demonstrates superior network performance for Bluetooth Low Energy networks.

Acknowledgments: This work was supported by the Ministry of Science and Technology, MOST 106-2221E-216-002 and MOST 106-2221-E-009-050, Taiwan.

Author Contributions: Chih-Min Yu designed and developed the multi-ring tree topology with the self-routing protocol. In addition, a min-path algorithm is introduced and simulated to determine the optimal MRT configuration for Bluetooth multi-hop scenario. He was also responsible for writing and revising the manuscript. Ting-Wei Hsu helped the validation stage of performance evaluation and simulation.

Conflicts of Interest: The authors declare no conflicts of interest.

\section{References}

1. With an Installed Base of 10 Billion Devices Expected in 2018, Bluetooth Will Be an Essential Tool for Building the Internet of Everything. Available online: https:/ /www.abiresearch.com/press/with-an-installed-baseof-10-billion-devices-expec/ (accessed on 15 July 2017).

2. Gupta, N.K. Inside Bluetooth Low Energy, 2nd ed.; Artech House: Norwood, MA, USA, 2013.

3. Etxaniz, J.; Gerardo, A. Modeling of the Data Transportation Network of a Multi-hop Data-content-sharing Home Network. IEEE Trans. Consum. Electron. 2015, 61, 31-38. [CrossRef]

4. Hung, C.H.; Bai, Y.W.; Tsai, R.Y. Design of blood pressure measurement with a health management system for the aged. IEEE Trans. Consum. Electron. 2012, 58, 619-625. [CrossRef]

5. Collotta, M.; Pau, G. An Innovative Approach for forecasting of Energy Requirements to improve a Smart Home Management System based on BLE. IEEE Trans. Green Commun. Netw. 2017, 1, 112-120. [CrossRef]

6. Methfessel, M.; Lange, S.; Kraemer, R.; Zessack, M.; Kollermann, P.; Peter, S. Real-life deployment of Bluetooth scatternets for wireless sensor networks. In Real-World Wireless Sensor Networks, Proceedings of the 5th International Workshop, REALWSN 2013, Como, Italy, 19-20 September 2013; Langendoen, K., Hu, W., Ferrari, F., Zimmerling, M., Mottola, L., Eds.; Springer: Berlin, Germany, 2014; pp. 43-51.

7. Leccese, F.; Cagnetti, M.; Sciuto, S.; Scorza, A.; Torokhtii, K.; Silva, E. Analysis, design, realization and test of a sensor network for aerospace applications. In Proceedings of the 2017 IEEE International Instrumentation and Measurement Technology Conference (I2MTC), Turin, Italy, 22-25 May 2017; pp. 1-6. [CrossRef]

8. Aziz, A.A.; Sekercioglu, Y.A.; Fitzpatrick, P.; Ivanovich, M. A Survey on Distributed Topology Control Techniques for Extending the Lifetime of Battery Powered Wireless Sensor Networks. IEEE Commun. Surv. Tutor. 2013, 15, 121-144. [CrossRef]

9. Hiew, Y.K.; Aripin, N.M.; Din, N.M. Performance of cognitive smart grid communication in home area network. In Proceedings of the 2014 IEEE 2nd International Symposium on Telecommunication Technologies (ISTT), Langkawi, Malaysia, 24-26 November 2014; pp. 417-422.

10. Chhaya, L.; Sharma, P.; Bhagwatikar, G.; Kumar, A. Design and Implementation of Remote Wireless Monitoring and Control of Smart Power System using Personal Area Network. Indian J. Sci. Technol. 2016, 9. [CrossRef]

11. Pau, G.; Collotta, M.; Maniscalco, V. Bluetooth 5 Energy Management through a Fuzzy-PSO Solution for Mobile Devices of Internet of Things. Energies 2017, 10, 992. [CrossRef] 
12. Whitaker, R.M.; Hodge, L.; Chlamtac, I. Bluetooth Scatternet Formation: A Survey. Ad Hoc Netw. 2005, 3, 403-450. [CrossRef]

13. Kamkuemah, M.; Le, H. A Study of Different Routing Protocols for Mobile Phone Ad Hoc Networks Connected via Bluetooth. In Proceedings of the 2013 UKSim 15th International Conference on Computer Modelling and Simulation, Cambridge, UK, 10-12 April 2013; pp. 681-686.

14. Al-Jarrah, O.; Megdadi, O. Enhanced AODV Routing Protocol for Bluetooth Scatternet. Comput. Electr. Eng. 2009, 35, 197-208. [CrossRef]

15. Sharafeddine, S.; Al-Kassem, I.; Dawy, Z. A Scatternet Formation Algorithm for Bluetooth Networks with a Non-uniform Distribution of Devices. J. Netw. Comput. Appl. 2012, 35, 644-656. [CrossRef]

16. Salonidis, T.; Bhagwat, P.; Tassiulas, L.; LaMaire, R. Distributed topology construction of Bluetooth personal area networks. IEEE J. Sel. Areas Commun. 2005, 23, 633-643. [CrossRef]

17. Yu, C.; Yu, Y. Reconfigurable Algorithm for Bluetooth Sensor Networks. Sensors J. IEEE 2014, 14, 3506-3507.

18. Zaruba, G.; Basagni, S.; Chlamtac, I. Bluetrees-scatternet formation to enable Bluetooth-based ad hoc networks. In Proceedings of the IEEE International Conference on Communications, Helsinki, Finland, 11-14 June 2001; Volume 1, pp. 273-277.

19. Chiasserini, C.F.; Marsan, M.A. A distributed self-healing approach to Bluetooth scatternet formation. IEEE Trans. Wirel. Commun. 2005, 4, 2649-2654. [CrossRef]

20. Li, X.Y.; Stojmenovic, I.; Wang, Y. Partial Delaunay triangulation and degree limited localized Bluetooth scatternet formation. IEEE Trans. Parallel Distrib. Syst. 2004, 15, 350-361. [CrossRef]

21. Petrioli, C.; Basagni, S.; Chlamtac, M. Configuring BlueStars: Multihop scatternet formation for Bluetooth networks. IEEE Trans. Comput. 2003, 52, 779-790. [CrossRef]

22. Tekkalmaz, M.; Sozer, H.; Korpeoglu, I. Distributed Construction and Maintenance of Bandwidth and Energy Efficient Bluetooth Scatternets. IEEE Trans. Parallel Distrib. Syst. 2006, 17, 963-974. [CrossRef]

23. Persson, K.E.; Manivannan, D.; Singhal, M. Bluetooth scatternets: Criteria, models and classification. Ad Hoc Netw. 2005, 3, 777-794. [CrossRef]

24. Yu, C.M.; Lin, E.L. Reliable Formation Protocol for Bluetooth Hybrid Single-hop and Multi-hop Networks. IEEE Netw. 2017, accepted.

25. Al-Karaki, J.N.; Kamal, A.E. Routing techniques in wireless sensor networks: A survey. IEEE Wirel. Commun. 2004, 11, 6-28. [CrossRef]

26. Guo, Z.; Harris, I.G.; Tsaur, L.F.; Chen, X. An on-demand scatternet formation and multi-hop routing protocol for ble-based wireless sensor networks. In Proceedings of the Wireless Communications and Networking Conference (WCNC), New Orleans, LA, USA, 9-12 March 2015; pp. 1590-1595.

27. Yu, C.M. Global Configured Method for Blueweb Routing Protocol. IET Commun. 2012, 6, 69-75. [CrossRef]

28. Sun, M.T.; Chang, C.K.; Lai, T.H. A self-routing topology for bluetooth scatternets. In Proceedings of the Parallel Architectures, Algorithms and Networks, Makati, Philippines, 22-24 May 2002; pp. 17-22.

29. Yu, C.M.; Chen, W.S.; Yu, Y.B. A Layer-Ring Formation Algorithm for Bluetooth Multi-hop Networks. In Proceedings of the 2014 International Symposium on Computer, Consumer and Control, Taichung, Taiwan, 10-12 June 2014; pp. 1082-1086.

30. Jeon, W.S.; Dwijaksara, M.H.; Jeong, D.G. Performance Analysis of Neighbor Discovery Process in Bluetooth Low-Energy Networks. IEEE Trans. Veh. Technol. 2017, 66, 1865-1871. [CrossRef] 